\title{
Fungal Isolates from a Marine Sponge from Dao Dao Island, Pagadian City against Bacillus subtilis, Microccocus luteus, Rhizopus oryzae, and Saccharomyces cerevisiae
}

Barbaso Pierre Angeli $\mathbf{P}^{\star}$, Lazaro SMB and Quiambao CKB

Philippine Science High School-Central Mindanao Campus, Nangka, Balo-i, Lanao del Norte, Phillipines

*Corresponding author: Barbaso PAP, Philippine Science High School-Central Mindanao Campus, Nangka, Balo-i, Lanao del Norte, Phillipines, Tel: 09196857369; E-mail: pierreangeli17@yahoo.com

Received: December 02, 2017; Accepted: December 20, 2017; Published: January 05, 2018

Copyright: () 2018 Barbaso PAP et al. This is an open-access article distributed under the terms of the Creative Commons Attribution License, which permits unrestricted use, distribution, and reproduction in any medium, provided the original author and source are credited.

\section{Abstract}

Scientists have probed into the diverse populations in the ecosystem searching for feasible solutions to the rising existence of antibiotic resistance mechanisms that are threatening the ability to treat infectious diseases. Marine sponges are recognized as the richest sources of new marine natural products and have been shown to contain associated bacteria and fungi which produce compounds exhibiting microbial activity. In the study, fungal isolates obtained from an unidentified marine sponge were tested against Bacillus subtilis, Micrococcus luteus, Rhizopus oryzae and Saccharomyces cerevisiae. Three strips of the sponge were placed in three test tubes containing yeast peptone glucose (YPG) broth and were incubated for 7 days at $37^{\circ} \mathrm{C}$. The three samples were each diluted in ten test tubes. One and a half $\mathrm{mL}$ was pipetted to YPG agar plates from test tubes 8 to 10 for every sample. Four fungal isolates were obtained from the three samples and purified through streak plate method. These were then assayed through cross streak method using modified YPG agar as the medium. Of the four isolates tested against the four test organisms, only one isolate exhibited activity against Rhizopus oryzae and none against the other organisms. The isolate was further tested through disc diffusion technique. Analysis of zones of inhibition proved that antifungal activity was exhibited. This provides another source of inhibitory compound. Further tests are recommended to validate results.
\end{abstract}

Keywords: Antifungal; Associated microorganisms; Sponge

\section{Introduction}

\section{Background of the study}

Numerous cases of diseases caused by the infection of viruses or bacteria are now being cured and prevented by a variety of drugs, antibiotics and vaccines. These have been the products of intensive research throughout the years. Scientists have probed into the diverse populations that make up the ecosystem, and searched for feasible solutions to the problems that human health faces today. However, new antibiotic resistance mechanisms are emerging and spreading globally, threatening the ability to treat common infectious diseases, resulting in prolonged illness, disability, and death [1]. This is further worsened by the fact that antibiotic drug discovery and development seem to have slowed down over the years [2]. Thus, the search for antibiotics is greatly encouraged and highly needed. Among the options of several possibilities for protection against pathogenic microorganisms, searching for new antibiotics is considered to be very important [3].

Natural products have historically been of crucial importance in the identification and development of antibacterial agents, but the rapid emergence of resistant bacterial strains has forced their re-evaluation as a route to identify novel chemical skeletons with antibacterial activity for elaboration in drug development [4]. Due to the great chemical variability provided by biological diversity existing in the marine environment, research in marine natural products has received great interest [5]. The discovery of marine natural products has accelerated over the last two decades with the number of new compounds discovered annually increasing from 20 to more than 200 [6].

Among all the marine organisms investigated, marine sponges (Porifera) are recognized as the richest sources of new marine natural products, with about 4851 compounds to date, contributing to nearly $30 \%$ of all marine natural products discovered so far [6]. These multicellular animals have more than 8,000 species [7] thriving along with other organisms in benthic communities. As these marine invertebrates are sessile and soft bodied, they rely on chemical defenses (also known as natural products), which arose through their evolutionary history to deter predators, to keep competitors away, or to paralyze prey [8]. Attempts to isolate these chemical compounds are now done for its utilization in medical research and development.

However, not only do these marine sponges exhibit antimicrobial activities, but they also contain associated bacteria that have been shown to exhibit similar antimicrobial activities. The majority of these compounds are thought to not be produced by the sponge itself, but rather by their associated bacteria or fungi [9]. A study conducted by Anand et al. [10] showed that out of the isolated 75 marine bacterial strains associated with four species of sponges, $21 \%$ were found to contain antibiotic properties. Other studies continued to focus on testing the antimicrobial properties of microbial isolates from marine sponges ever since a noteworthy similarity was found between the bioactive compounds isolated from sponges and those from a terrestrial organism from completely different taxa [11]. 
Citation: Barbaso PAP, Lazaro SMB, Quiambao CKB (2018) Fungal Isolates from a Marine Sponge from Dao Dao Island, Pagadian City against Bacillus subtilis, Microccocus luteus, Rhizopus oryzae, and Saccharomyces cerevisiae. Nat Prod Chem Res 6: 309. doi:

Page 2 of 10

In this study, the researchers tested fungal isolates obtained from a sponge species in Dao Dao Island, Pagadian City, against Bacillus subtilis, Micrococcus luteus, Rhizopus oryzae and Saccharomyces cerevisiae. A study on sponges by Pastoriza and Sigod [12] has been conducted in the same area. However, the study investigated the crude extracts from a marine sponge, whereas this study examined the activity of microbial isolates from a marine sponge. Identification of the sponge species from which the microbial isolates were extracted, was not carried out in the study.

The presence of great amounts of bacteria is distinctive for demosponges-sponges that belong to the most diverse class in phylum Porifera; Demospongiae [13]. A. aerophoba has been shown by Friedrich et al. to still contain its microbial community after being treated with antibiotics. Hausmann et al. [13] also stated that bacteria are found on the sponge surface, in its canal system, in its intracellular matrix, or in the outer parts of the tissue. This suggests that its associated bacteria are also cultivated along with the growth of the species in the laboratory.

Different species of marine sponges have been explored by other researchers globally, and their bioactivity has been studied. However, the conduct of related studies in areas that have not yet been fully explored is necessary since a variation in location might yield a different array of compounds [14].

\section{Objectives of the study}

This study aims to determine which among the fungal isolates from a marine sponge exhibit anti-microbial activity against selected microorganisms. Specifically, it aims to:

1. Isolate and obtain pure colonies of associated fungi from a marine sponge from Dao Dao Island, Pagadian City; and

2. Investigate anti-microbial activity of fungal isolates against the gram-positive bacteria, Bacillus subtilis and Micrococcus luteus, the mold, Rhizopus oryzae, and the yeast, Saccharomyces cerevisiae.

\section{Significance of the study}

The discovery of new sources of antimicrobial compounds would be very helpful as the need nowadays is rising. The addition of new sources for potentially inhibiting substances would be very beneficial for medical development, as it would contribute to the existing studies exploring biochemical processes in marine inhabitants. Furthermore, conducting the study in a rarely explored environment allows for improvement and further succeeding justifications for the studies conducted on marine sponge microbial isolates. This study also offers new information on possible outcomes of geographical differences in sponge species and the compounds that are produced by its associated microorganisms.

\section{Scope and limitations of the study}

This study is limited to the testing for the presence of inhibiting potential in fungal isolates obtained from a marine sponge in Dao Dao Island, Pagadian City. However, for those isolates that may exhibit positive inhibiting activity, identification and isolation of its specific chemical compounds is not included in this study. The degree of inhibition is compared to nystatin, the positive control. Ethyl acetate is used as the negative control. Results are treated primarily as additional experimental data for the development of medical research. The experiments that are conducted only encompass selected representatives from bacteria and fungi.

\section{Definition of terms}

- Benthic-relating to, or occurring at the bottom of a body of water.

- Bioactive-Having or producing an effect on living tissue.

- Extant-Still existing.

- Inoculation-The introduction of a pathogen or antigen into a living organism to stimulate the production of antibodies.

\section{Review of related literature}

Mankind has known for the last several thousand years that marine organisms contain substances capable of potent biological activity [15]. Due to the complex and intricate nature of the ocean, marine bacteria have developed sophisticated physiological and biochemical systems with which they uniquely adapt to extreme habitats and various unfavorable marine environmental conditions [16]. High competition in the ecosystem is also a factor of the development of biological defense systems by physically weak organisms to be able to protect themselves [17].

Sponges are considered as the chemical factory in marine environment because of its immense production of chemically diverse compounds [18]. Many studies concerning the bioactivity of sponges have been conducted, extending explorations to the extraction and isolation of secondary metabolites. Bioactive substances from sponges or associated microorganisms have shown broad spectrum properties such as anticancer, antibacterial, antifungal, antiviral, antiprotozoal, anthelmintic, anti-inflammatory, immunosuppressive, neurosuppressive and antifouling [19]. It is suggested that (at least) some of the bioactive secondary metabolites isolated from sponges are produced by functional enzyme clusters, which originated from the sponges and their associated microorganisms [15]. Secondary metabolites-bioactive compounds generated majorly from marine animals-are organic compounds that are not directly involved in the normal growth, development, or reproduction of an organism [15].

These secondary metabolites are now used as suppressants in numerous diseases worldwide. In the study of Sinko et al. [19], it was clearly stated that the natural products from wreath lichen (Cryptotheca) served as a template for the synthesis of the anti-viral drug Ara-A that led to the development of the medicinal drug for AIDS. Although these are terrestrial species, it has been stated that there has been a remarkable similarity between compounds produced by organisms in land and on water and should therefore put forward that secondary metabolites isolated from both areas have equally great potential for medical uses. The potential of marine sponges in Zanzibar to yield antimalarial and antimicrobial compounds have also been explored by Said et al. [20]. Tests were run against the fungi Candida albicans and Crypotococcus neoformans where high activity was exhibited by ethyl acetate extracts of Agelas mauritania and Oceanopia sp.

Marine sponges Biemna tubulosa and Stylissa spp. collected from a lagoon in Mauritius have also been reported to have antibacterial activity by Soulange et al. [21]. Crude extracts and fractions of these marine sponges were tested against the bacterial organisms Escherichia coli, Staphylococcus aureus, and Enterococcus faecalis. It was found that the most non-polar extracts of Biemna tubulosa and polar extracts 
Citation: Barbaso PAP, Lazaro SMB, Quiambao CKB (2018) Fungal Isolates from a Marine Sponge from Dao Dao Island, Pagadian City against Bacillus subtilis, Microccocus luteus, Rhizopus oryzae, and Saccharomyces cerevisiae. Nat Prod Chem Res 6: 309. doi: 10.4172/2329-6836.1000309

of Stylissa spp. had higher antimicrobial activity than the reference antibiotic, Chloramphenicol.

In the study of Dingal [14], Thorecta sp., cream column sponge, collected from Misamis Occidental, Philippines, was homogenized in methanol, and was shown to exhibit clearing activities against Staphylococcus aureus and Bacillus subtilis. The crude extracts from the sponge species also showed minimum inhibitory concentration (MIC) on Pseudomonas aeruginosa, Escherichia coli, Bacillus subtilis, and Aspergillus niger at $125 \mathrm{ppm}$ and on Staphylococcus aureus at 1.95 ppm. The choloroform-methanol (3:1) solvent system was used in Thin Layer Chromatography (TLC), producing four unidentified fractions with $R_{f}$ values of $0.96,0.91,0.52$, and 0.31 . Dingal [14] proved that Thorecta sp. is a potential broad-spectrum antibiotic comparable to commercial standards, Chloramphenicol and Nystatin.

Pastoriza and Sigod [12] also showed that an unidentified sponge collected from White Sand Island, Pagadian City, also known as Dao Dao Island, had a significant inhibitory effect on $E$. coli, $P$. aeruginosa, $S$. aureus and $B$. cereus. The extracts at three different concentrations exhibited zones of inhibition of $30.89 \mathrm{~mm}, 23.11 \mathrm{~mm}$, and $21.22 \mathrm{~mm}$ activity significantly greater than the commercial standard Chloramphenicol which exhibited zones of inhibition of $18.00 \mathrm{~mm}$, $13.00 \mathrm{~mm}, 17.00 \mathrm{~mm}$, and $14.00 \mathrm{~mm}$.

The different studies mentioned above concerning the bioactivity of compounds from marine sponges and its associated microorganisms showed that these organisms could be promising sources of antibiotic substances. There is already a wide selection of studied marine sponges containing potential antibiotic sources. International and local studies have contributed to the growing range of data in the field. However, further studies are still important to verify the existing data, discover new sources, and provide more evidences to prove the potential and viability of the compounds extracted.

\section{Methodology}

\section{Sampling area}

Samples were collected from the Dao Dao Island, Pagadian City (Figures 1 and 2). The sampling site was a 30 -minute motorboat ride from the Pagadian City port (Figure 3 ).

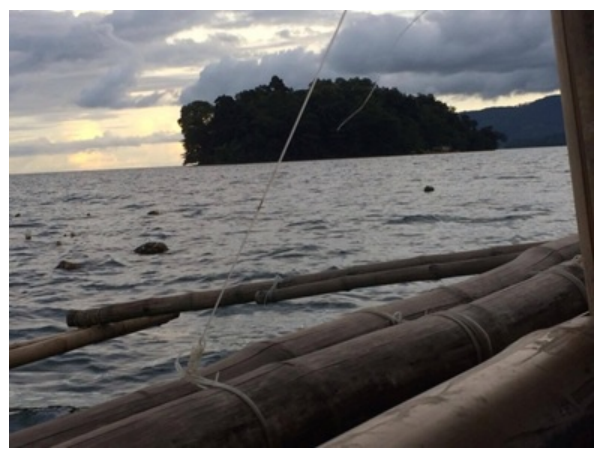

Figure 1: Dao Dao Island from afar (Photo taken January 5, 2017, Pagadian City).

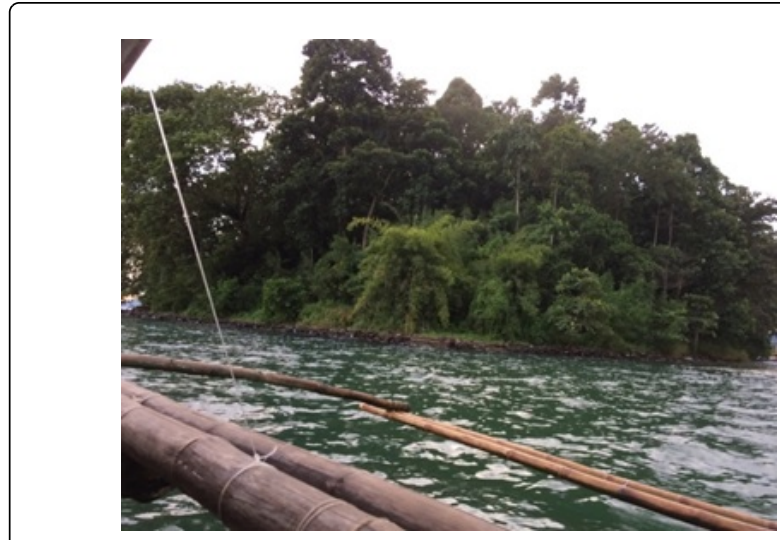

Figure 2: Dao Dao Island, approximately 15 meters from land (Photo taken January 5, 2017, Pagadian City).

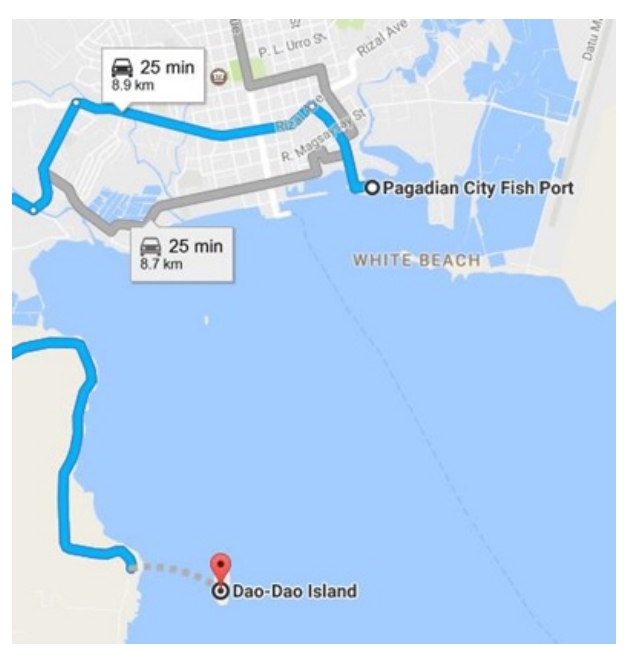

Figure 3: Location of Dao Dao Island, Pagadian City (Google Maps, 2017).

\section{Collection of sponges}

Sponge samples were collected at 3-5 meters depth of salt water surrounding the Dao Dao Island. Photographs were taken to aid in species identification (Figure 4). Only a portion of the sponge body was cut by a knife and collected to enable the sponge to regenerate [14]. The cut portion was placed in a zipped-lock bag underwater [22]. At the sampling site, the sponge sample was washed with sterilized seawater. Thorough washing was done in order to carefully remove contaminants such as sediments and sand. Further washing was done using sterilized distilled water [12]. 
Citation: Barbaso PAP, Lazaro SMB, Quiambao CKB (2018) Fungal Isolates from a Marine Sponge from Dao Dao Island, Pagadian City against Bacillus subtilis, Microccocus luteus, Rhizopus oryzae, and Saccharomyces cerevisiae. Nat Prod Chem Res 6: 309. doi: $10.4172 / 2329-6836.1000309$

Page 4 of 10

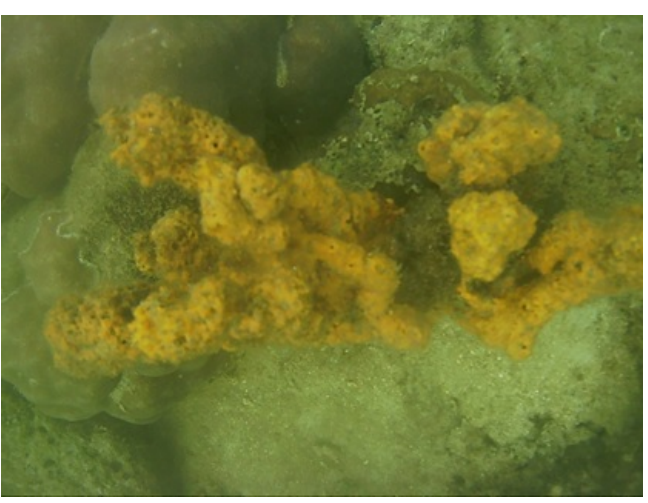

Figure 4: Unidentified marine sponge (Photo taken November 12, 2016, Dao Dao Island, Pagadian City).

\section{Culturing fungi from samples}

Three test tubes were prepared containing yeast peptone glucose (YPG) broth see Appendix. Employing strict aseptic techniques, the sponge sample was cut into thin strips with sterile scissors about $1 / 2 \mathrm{~cm}$ $\times 1 \mathrm{~cm} \times 6 \mathrm{~cm}$, and were washed again with sterilized distilled water. One strip from the sponge sample was immersed in each of the test tubes. These were then labelled based on the media and were incubated at $37^{\circ} \mathrm{C}$ for 4 days. This length of time contributed to the sufficient growth of the fungi of interest [23].

\section{Appendix}

Preparation and ingredients of media and reagents:

C.1. Yeast Peptone Glucose Broth.

Yeast Extract-20 g

Peptone-20 g

Glycerine-30 mL

\section{Distilled Water-1 L}

Suspend 20 grams each of yeast extract and peptone in $1000 \mathrm{~mL}$ distilled water containing $30 \mathrm{~mL}$ Glycerine. Heat to boiling to dissolve the medium completely. Sterilize by autoclaving at $15 \mathrm{lbs}$ pressure $\left(121^{\circ} \mathrm{C}\right)$ for 15 minutes [24].

\section{C.2. Yeast peptone glucose agar (HiMedia Laboratories)}

Yeast Extract-10 g

Peptone-20 g

Agar Powder-20 g

Glycerine-30 mL

Distilled Water-1 L

Suspend 50 grams in $1000 \mathrm{~mL}$ distilled water containing $30 \mathrm{~mL}$ glycerine. Heat to boiling to dissolve the medium completely. Sterilize by autoclaving at $15 \mathrm{lbs}$ pressure $\left(121^{\circ} \mathrm{C}\right)$ for 15 minutes [25].

\section{C.3. Natural saline solution (0.6\%) (Tag My Doc)}

Sodium Chloride- $0.6 \mathrm{~g}$

\section{Distilled Water-100 mL}

Make certain all apparatus is clean, dry, and sterile [26]. This is to prevent contamination from the solution and also to avoid dilution from the preparation. Inside a clean analytical balance, weigh 0.85 grams of sodium chloride. Make sure to subtract the load from the container to get the proper weight of sodium chloride. After weighing, get yourself a $100 \mathrm{~mL}$ adjusted volumetric flask, and pipet $50 \mathrm{~mL}$ in to the volumetric flask. Carefully transfer the powder/deposits using a spatula towards the volumetric flask using the $50 \mathrm{~mL}$ of sterilized water. Dissolve the powder by mixing lightly having a stirring fishing rod. Add sterilized water towards the volumetric flask as much as the $100 \mathrm{~mL}$ mark. Stir again until all of the powder continues to be dissolved. Transfer to some clean, dry and sterile storage bottle and label correctly. Should you prefer a bigger volume, all you need to do would be to solve for that weight in grams of sodium chloride needed through ratio and proportion $(0.85 \times 1,500 \mathrm{~mL} / 100 \mathrm{~W}=12.75 \mathrm{~g})$.

\section{C.4. Top agar: Bado}

\begin{tabular}{|l|l|}
\hline Sodium Chloride & $0.6 \mathrm{~g}$ \\
\hline Agar Powder & $0.5 \mathrm{~g}$ \\
\hline Distilled Water & $100 \mathrm{~mL}$ \\
\hline
\end{tabular}

Ingredients were dissolved in distilled water and were sterilized in an autoclave for 15 minutes at $121^{\circ} \mathrm{C}$. After sterilization, the dissolved components were cooled at a temperature of about $45^{\circ} \mathrm{C}$ [23].

\section{Obtaining fungal colonies from sponge}

The three test tubes containing the strips of the marine sponge were then subjected to serial dilution and pour plate technique [23]. Ten test tubes per sample were used, each test tube containing nine $\mathrm{mL}$ of $0.6 \%$ Natural Saline Solution (NSS) [23]. For every ten test tubes, these were labelled with numbers 1 to 10. Employing strict aseptic techniques, one and one-half $\mathrm{mL}$ of aliquot was pipetted from the first test tube containing a strip of the sample and was dispensed to test tube 1 . The tube was homogenized thoroughly, and then another one and one-half $\mathrm{mL}$ of aliquot was pipetted and dispensed to the second tube up to the tenth tube, following the serial dilution [24]. From test tubes 8 to 10, one and one-half $\mathrm{mL}$ of aliquot was pipetted and dispensed to the corresponding YPG agar plates with three plates for each tube. This was done for the three test tubes containing the sample sponge strips. The plates were then incubated for 7 days at $37^{\circ} \mathrm{C}$.

\section{Purification of fungal isolates by streak plate method}

From the incubated petri dishes, distinct colonies were selected based on elevation, surface, and color. An inoculation loop was used to collect and transfer these cultured colonies from the petri dish for streak plate method. Colonies were transferred to another petri dish containing YPG agar by doing the four quadrant streak. The plates were then incubated for 48 hours at a temperature of $37^{\circ} \mathrm{C}$. This method was repeated until purified fungal isolates were acquired.

\section{Testing for antimicrobial activity against selected microorganisms by cross streak method}

The isolates obtained through streak plate determined the number of fungal colonies to be tested using generally non-pathogenic test organisms, namely: Bacillus subtilis, Micrococcus luteus, Rhizopus 
Citation: Barbaso PAP, Lazaro SMB, Quiambao CKB (2018) Fungal Isolates from a Marine Sponge from Dao Dao Island, Pagadian City against Bacillus subtilis, Microccocus luteus, Rhizopus oryzae, and Saccharomyces cerevisiae. Nat Prod Chem Res 6: 309. doi: $10.4172 / 2329-6836.1000309$

Page 5 of 10

oryzae and Saccharomyces cerevisiae. The four test organisms were seeded in modified yeast peptone glucose (YPG) plates (i.e., 50\% YPG agar and $50 \%$ nutrient agar). This modification was done to ensure the growth of fungal isolates and the fungal and bacterial test organisms [23]. Ten $\mathrm{ml}$ from the sterilized media was poured on separate sterile plates and were allowed to solidify. To ensure uniformity of inoculum in each assay plate, top agar technique was employed [24]. This was prepared in the laboratory.

The procedure was patterned after Bado [23]. The top agar was prepared by dissolving $0.5 \mathrm{~g}$ of agar and $0.6 \mathrm{~g}$ of $\mathrm{NaCl}$ in $100 \mathrm{~mL}$ of distilled water and was autoclaved at $121^{\circ} \mathrm{C}$ for 15 minutes. After the top agar was cooled down to around $40^{\circ} \mathrm{C}$, one loopful of inoculum of each test organism was aseptically added and then thoroughly mixed to facilitate even distribution. Two and a half milliliter of the seeded top agar was pipetted into each of the agar plates. The inoculum was spread over by the slight rotation of the plate. The plate was then labelled respectively to the kind of test organism used and was allowed to solidify.

Each isolate was tested against the four test organisms. Using an inoculating loop, an inoculum was obtained from an isolate and was streaked three times (parallel to each other, about $15 \mathrm{~mm}$ apart, see Figures 5 and 6 , for every plate previously seeded with a test organism. A total of 16 plates were then incubated for 48 hours at $37^{\circ} \mathrm{C}$.

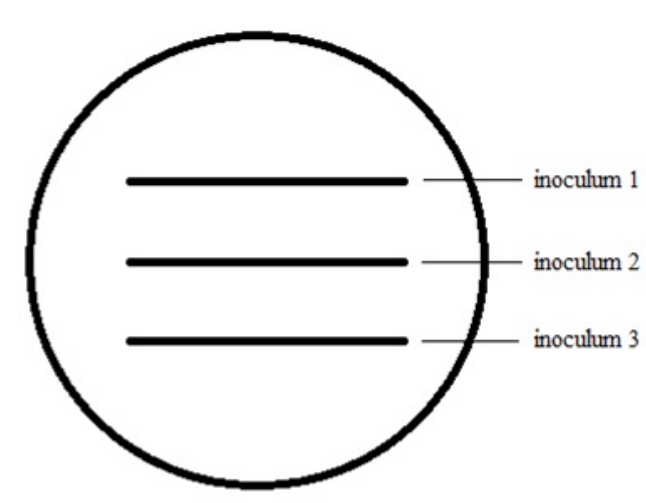

Figure 5: Illustration for inoculation for cross streak method.

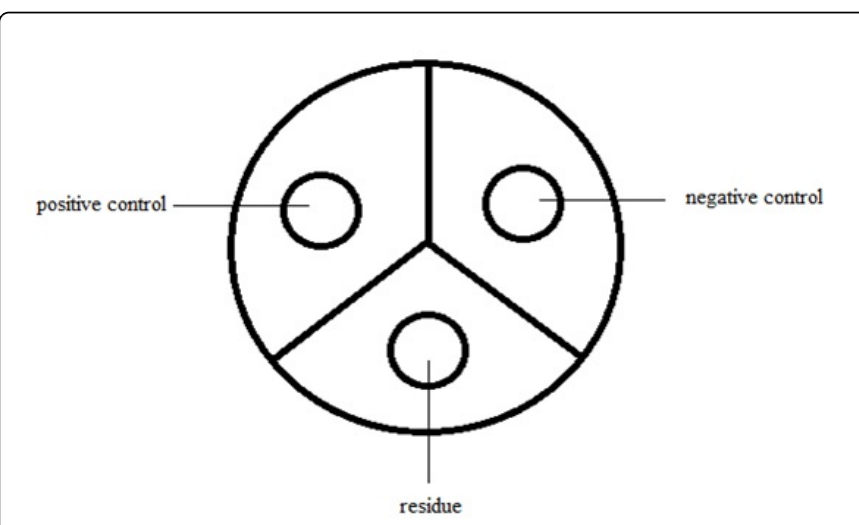

Figure 6: Illustration of the placement of filter paper discs for disc diffusion technique.

\section{Extraction of active components}

The fungal isolate that exhibited clearing zones was purified again by streak plate method, and was stocked in slant test tubes containing YPG agar [14]. An inoculum was obtained from the slant test tubes and was transferred to test tubes containing $10 \mathrm{~mL}$ of YPG broth. These were then poured in separate petri dishes containing YPG agar and incubated for 48 hours. The purified isolates were then macerated using a small mesh strainer and submerged in a beaker of ethyl acetate with a ratio of $2: 1$, to obtain the components of the fungi. This was then covered and allowed to set for 24 hours. After, the mixture was filtered through a funnel with filter paper to collect the filtrate that was later subjected to rotary evaporation; the process acquiring the crude extract.

\section{Disc diffusion technique}

Each sterile filter paper disc was impregnated aseptically with 15 microliters of the extract and the standard controls using a micropipette [23]. The plate was then divided into three sectors; one for nystatin which was the positive control, one sector for the negative control, and one sector for the crude extract. The negative control used in the experiment was ethyl acetate, as this was found to exhibit no activity or zone of inhibition as tested by Mustanir et al. [25]. Each impregnated disc was allowed to dry before being placed accordingly on the center of each sector. The plates were then incubated for 48 hours at $37^{\circ} \mathrm{C}$. The diameter of the zone of inhibition was measured in millimeters using a clear ruler.

\section{Statistical analysis of gathered data}

One-Way ANOVA was used in the analysis of the means of zones of inhibition with respect to the test fungi to determine if there was a significant difference among the zones of inhibition exhibited by the treatments $[12,14]$. The comparison of the significant means was done using Tukey Honestly Significant Difference (HSD) Test. Alpha ( $\alpha$ ), the level of significance value, was set at 0.05 rejection level considering that the study dealt with the potential anti-microbial activity from the sponge fungal isolates.

In the statistical analysis, the null hypothesis (Ho) of this study was (Hoa): there was no significant difference between the reactions of fungal isolates against the test organisms in terms of inhibition zones; and (Hob): there was no significant difference between the zones of inhibition exhibited by the fungal isolates compared to the control standards. The alternative hypothesis (H1) stated otherwise.

The hypothesis that was accepted was determined using a decision criterion:

(1) If $\mathrm{F}_{\text {computed }}>\mathrm{F}_{\text {tabulated, }}$, reject the null hypothesis, Ho, thus, accept the alternative hypothesis;

(2) If $\mathrm{F}_{\text {computed }}<\mathrm{F}_{\text {tabulated }}$, accept the null hypothesis, Ho, thus, reject the alternative hypothesis.

Cluster analysis was used to show the percentage of similarity between the average zones of inhibition exhibited by each treatment, and the distance matrix. The statistical analysis was aided by the $\mathrm{R}$ Statistical Software [26] and the PAST Software [27-30]. 
Citation: Barbaso PAP, Lazaro SMB, Quiambao CKB (2018) Fungal Isolates from a Marine Sponge from Dao Dao Island, Pagadian City against Bacillus subtilis, Microccocus luteus, Rhizopus oryzae, and Saccharomyces cerevisiae. Nat Prod Chem Res 6: 309. doi: 10.4172/2329-6836.1000309

Page 6 of 10

\section{Results and Discussion}

Four pure isolates were obtained from the fungal colonies grown on YPG agar by streak plate method. Preliminary examination for antibacterial activity against Bacillus subtilis and Micrococcus luteus, and anti-fungal activity against Rhizopus oryzae and Saccharomyces cerevisiae was conducted.
Results show (Table 1) that only isolate 2 exhibits anti-fungal activity against the mold Rhizopus oryzae see Figures 7-10. However, this isolate did not exhibit any clearing zone against the three other test organisms. All the other isolates did not exhibit any activity against the four test organisms.

\begin{tabular}{|l|l|l|l|l|}
\hline & \multicolumn{4}{|c|}{ Test Organisms } \\
\hline & Bacillus subtilis & Micrococcus luteus & Rhizopus oryzae & Saccharomyces cerevisiae \\
\hline Isolate 1 & 0 & 0 & 0 & 0 \\
\hline Isolate 2 & 0 & 0 & 1 & 0 \\
\hline Isolate 3 & 0 & 0 & 0 & 0 \\
\hline Isolate 4 & 0 & 0 & 0 & 0 \\
\hline 0=no detectable activity, 1=positive activity. & & & \\
\hline
\end{tabular}

Table 1: Anti-microbial Activity of Fungal Isolates.

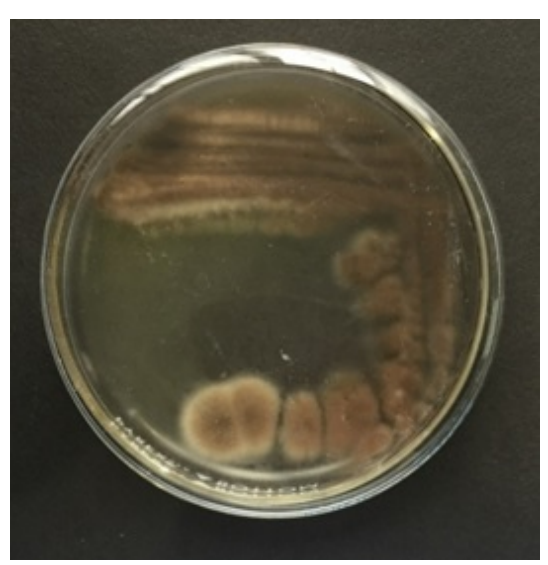

Figure 7: Fungal isolate 1-raised, wrinkled, and brown (Photo taken March 6, 2017, Biology Laboratory, PSHS-CMC).

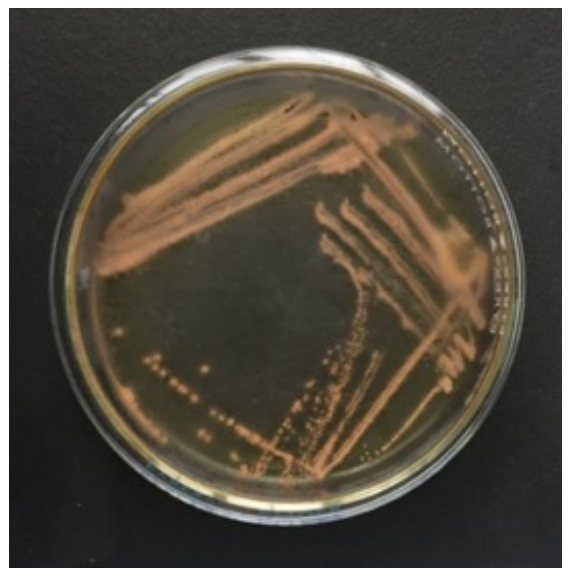

Figure 8: Fungal isolate 2-flat, smooth, and orange (Photo taken February 6, 2017, Biology Laboratory, PSHS-CMC). 
Citation: Barbaso PAP, Lazaro SMB, Quiambao CKB (2018) Fungal Isolates from a Marine Sponge from Dao Dao Island, Pagadian City against Bacillus subtilis, Microccocus luteus, Rhizopus oryzae, and Saccharomyces cerevisiae. Nat Prod Chem Res 6: 309. doi: $10.4172 / 2329-6836.1000309$

Page 7 of 10

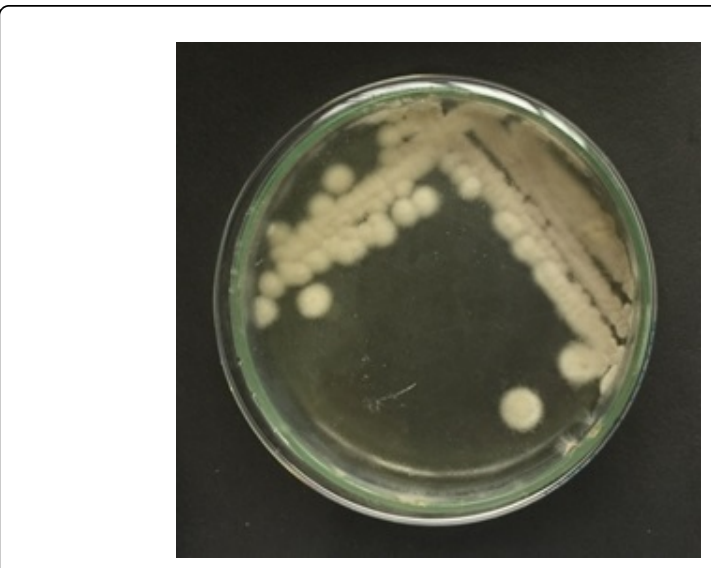

Figure 9: Fungal isolate 3-raised, wrinkled, and white (Photo taken March 6, 2017, Biology laboratory, PSHS-CMC).

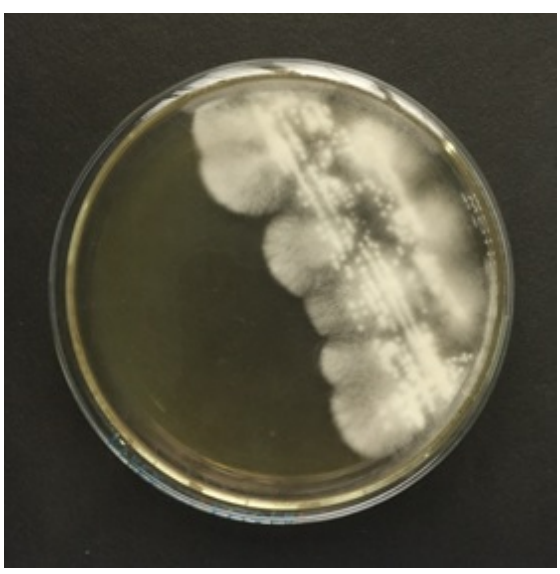

Figure 10: Fungal isolate 4-raised, cottony, and white (Photo taken March 6, 2017, Biology Laboratory, PSHS-CMC).

There is an evident competition between the isolate and the test organism in acquiring resources present in the medium. Clearing zones shown by isolate 2 against Rhizopus oryzae (Figures 11-16) may have been due to the isolate's ability to acquire more nutrients from the medium than the mold did. Furthermore, the isolate has exhibited positive activity against the fungus. Less growth of Rhizopus oryzae was observed around the three streaks of the isolate suggesting that there had been inhibition of mold growth.

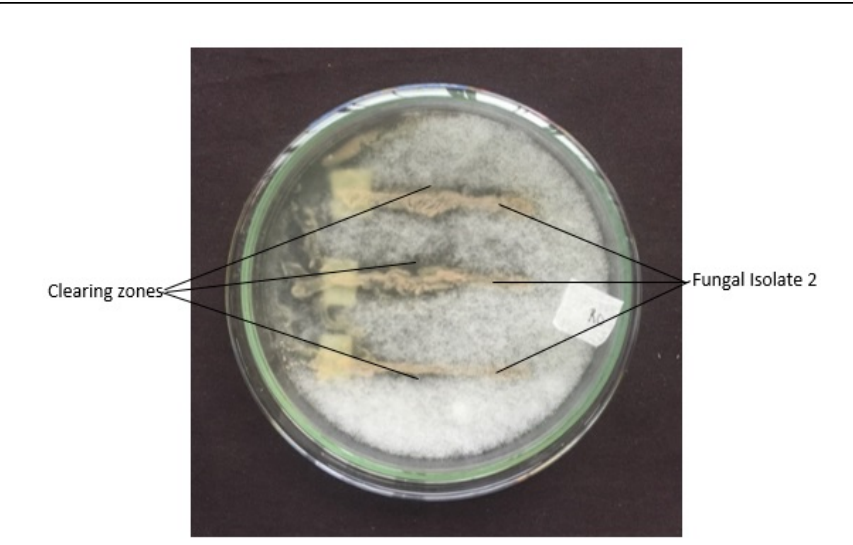

Figure 11: Clearing zones exhibited by the fungal isolate 2 against Rhizopus oryzae by cross streak method (Photo taken March 10, 2017, Biology Laboratory, PSHS-CMC).

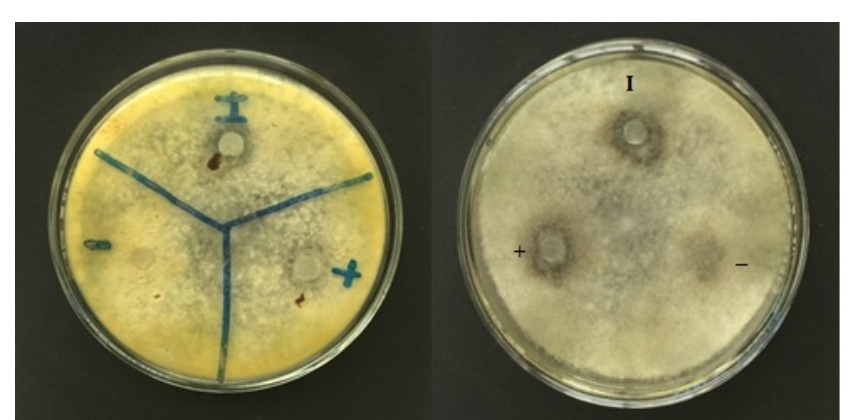

Figure 12: Testing for antifungal activity against Rhizopus oryzae by disc diffusion method (Trial 1 Replicate 1) (Photo taken May 2, 2017, Biology Laboratory, PSHS-CMC).

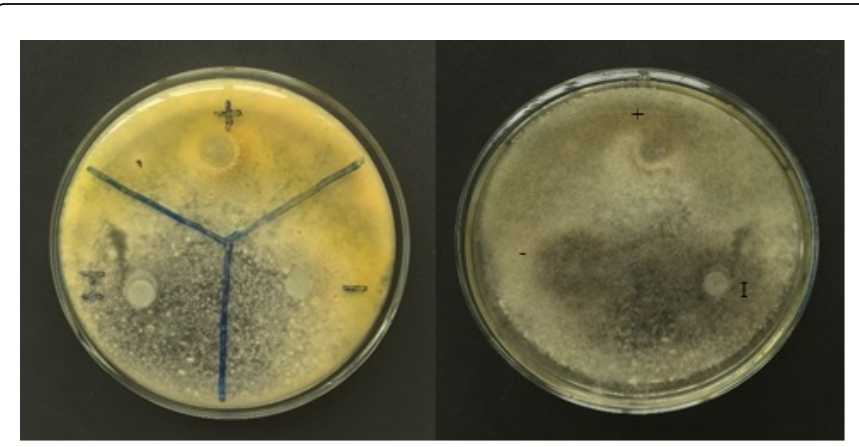

Figure 13: Testing for antifungal activity against Rhizopus oryzae by disc diffusion method (Trial 2 Replicate 1) (Photo taken May 2, 2017, Biology Laboratory, PSHS-CMC). 
Citation: Barbaso PAP, Lazaro SMB, Quiambao CKB (2018) Fungal Isolates from a Marine Sponge from Dao Dao Island, Pagadian City against Bacillus subtilis, Microccocus luteus, Rhizopus oryzae, and Saccharomyces cerevisiae. Nat Prod Chem Res 6: 309. doi: $10.4172 / 2329-6836.1000309$

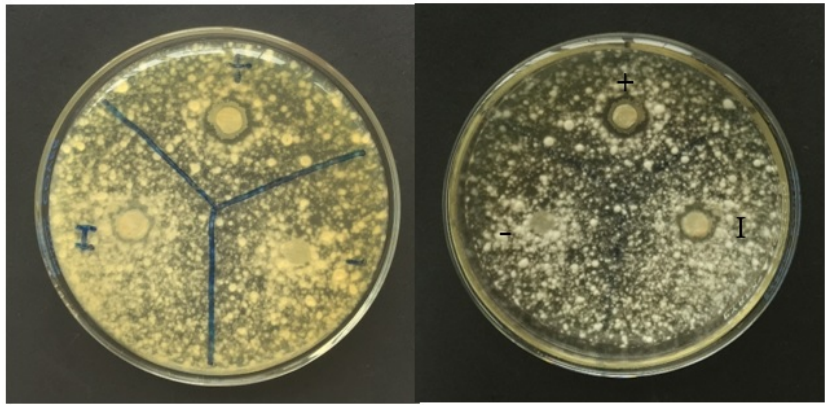

Figure 14: Testing for antifungal activity against Rhizopus oryzae by disc diffusion method (Trial 3 Replicate 1) (Photo taken May 2, 2017, Biology Laboratory, PSHS-CMC).

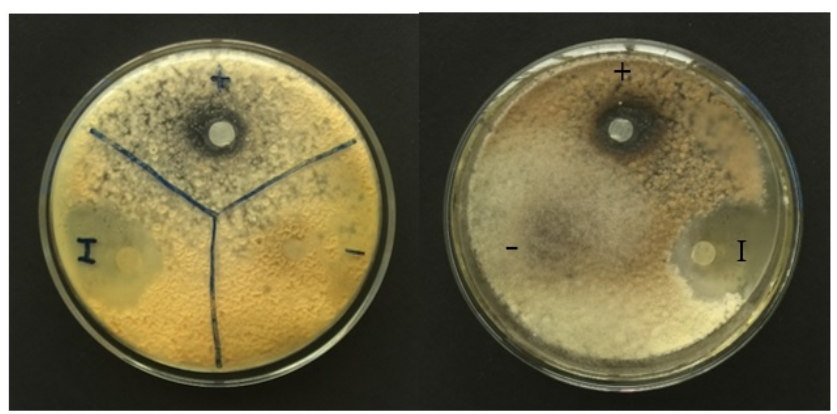

Figure 15: Testing for antifungal activity against Rhizopus oryzae by disc diffusion method (Trial 3 Replicate 2) (Photo taken May 2, 2017, Biology Laboratory, PSHS-CMC).

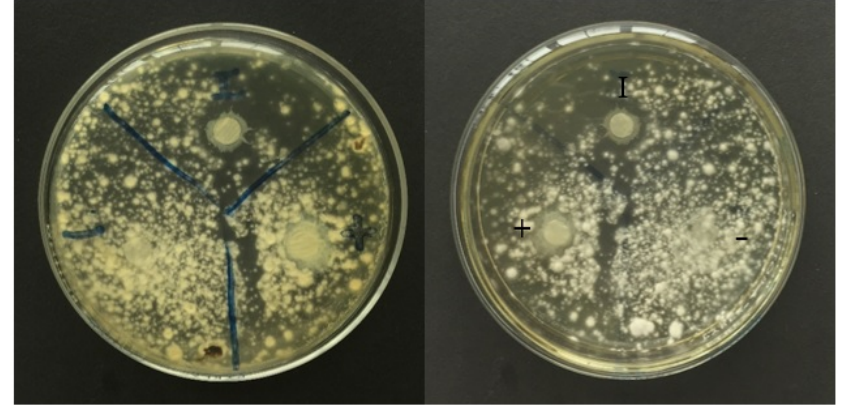

Figure 16: Testing for antifungal activity against Rhizopus oryzae by disc diffusion method (Trial 4 Replicate 1) (Photo taken May 2, 2017, Biology Laboratory, PSHS-CMC).

Results of the disc diffusion test show that out of the twelve replicates done in four separate trials, the isolate exhibited a zone of inhibition against Rhizopus oryzae in only five replicates (Table 2). This implies that the isolate contains antifungal compounds that may potentially be used against the test organism.

\begin{tabular}{|l|l|l|l|l|l|l|l|l|l|l|l|l|}
\hline & \multicolumn{3}{|l}{ Trial 1 } & \multicolumn{3}{l}{ Trial 2 } & \multicolumn{3}{l|}{ Trial 3 } & \multicolumn{2}{l|}{ Trial 4 } \\
\cline { 2 - 13 } & R1 & R2 & R3 & R1 & R2 & R3 & R1 & R2 & R3 & R1 & R2 & R3 \\
\hline Isolate & 0 & 12 & 0 & 10 & 0 & 0 & 10 & 30 & 0 & 14 & 0 & 0 \\
\hline Nystatin & 11 & 11 & 19 & 10 & 10 & 10 & 12 & 17 & 14 & 14 & 14 & 14 \\
\hline Ethyl Acetate & 0 & 0 & 0 & 0 & 0 & 0 & 0 & 0 & 0 & 0 & 0 \\
\hline
\end{tabular}

Table 2: Zones of Inhibition Exhibited by the Isolate Against Rhizopus oryzae (mm).

Using the Paleontological Statistics (PAST) software, one-way analysis of variance (ANOVA) with a 0.05 level of significance was carried out. Results showed a p value of 0.00109 (Table 3). This suggests that there is a significant difference between the means of zones of inhibition exhibited by the isolate, nystatin, and ethyl acetate, implying that the isolate exhibits antifungal activity against the test organism.

\begin{tabular}{|l|l|l|l|l|l|}
\hline & $\mathbf{d F}$ & $\begin{array}{l}\text { Sum of } \\
\text { Sq }\end{array}$ & Mean Sq & F value & $\operatorname{Pr}(>\mathbf{F})$ \\
\hline Treatment & 2 & 667.7 & 333.9 & 12.7 & 0.00109 \\
\hline
\end{tabular}

\begin{tabular}{|l|l|l|l|l|l|}
\hline Residuals & 12 & 315.6 & 26.3 & - & - \\
\hline
\end{tabular}

Table 3: One-way ANOVA Results of the Mean Zones of Inhibition Exhibited by the Isolate, Nystatin (Positive Control), and Ethyl Acetate (Negative Control) Against Rhizopus oryzae.

Results of the Tukey's Pairwise Comparison of the $\mathrm{p}$ value of the zones of inhibition exhibited by the three treatments show that both nystatin and the isolate's $p$ values have a significant difference compared to that of ethyl acetate $(\operatorname{Pr}(>|t|)=0.00501,0.00134)$, and none compared to each other $(\operatorname{Pr}(>|t|)=0.74512)$ (Table 4). This implies that the isolate exhibits similar antifungal property against the test organism as the positive control. 
Citation: Barbaso PAP, Lazaro SMB, Quiambao CKB (2018) Fungal Isolates from a Marine Sponge from Dao Dao Island, Pagadian City against Bacillus subtilis, Microccocus luteus, Rhizopus oryzae, and Saccharomyces cerevisiae. Nat Prod Chem Res 6: 309. doi: 10.4172/2329-6836.1000309

Page 9 of 10

\begin{tabular}{|l|l|l|l|}
\hline & Isolate & Nystatin & Ethyl Acetate \\
\hline Isolate & - & 0.74512 & 0.00134 \\
\hline Nystatin & - & - & 0.00501 \\
\hline
\end{tabular}

Table 4: Tukey's Pairwise Comparisons of the P Value of the Zones of Inhibition Exhibited by the Isolate, Nystatin (Positive Control), and Ethyl Acetate (Negative Control) Against Rhizopus oryzae.

Figure 16 shows that the zones of inhibition exhibited by the isolate have a higher percentage of similarity with that of nystatin or the positive control. This implies that the isolate contains factors that exhibit antifungal properties significantly similar to nystatin, and that the isolate may contain feasible compounds which may be extracted and used for medicinal purposes. The compounds could be extracted and separated using different methods such as High Performance Liquid Chromatography (HPLC) and tested further for their individual capabilities. Thus, further studies would have to be conducted.

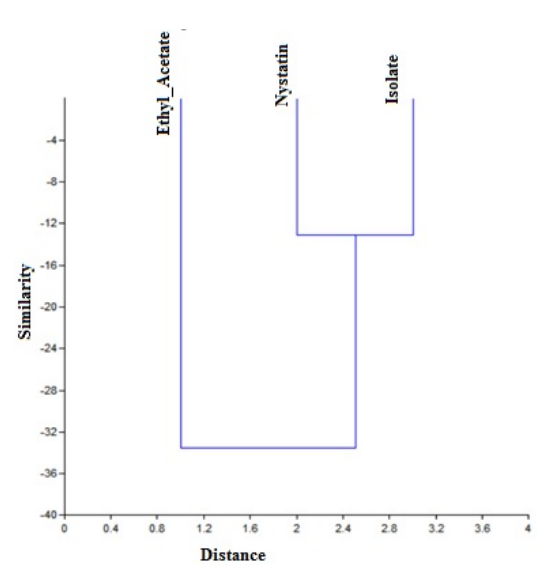

Figure 16: Cluster analysis showing the similarities of the treatments against Rhizopus oryzae.

In this study, only the fungi were isolated from the marine sponge collected from Dao Dao island. However, marine sponges are also known to harbor bacteria. Further studies could also be conducted wherein bacteria from the marine sponge would be isolated and also tested for antimicrobial activity.

\section{Conclusion and Recommendations}

One fungal isolate has shown positive activity against Rhizopus oryzae as seen after conducting the cross streak method. Clearing zones were exhibited throughout each streak. Rhizopus oryzae showed less growth around the streak indicating minimal regrowth after 48 hours of incubation. Statistical analysis of results from the disc diffusion technique show that both the isolate and nystatin exhibit significantly similar activity against Rhizopus oryzae. This suggests that the fungal isolate could be a potential source of an inhibitory compound.

It is recommended that further tests such as HPLC be used to obtain the pure extract of active compounds. To contribute to the growing knowledge in medical research, testing the antimicrobial activity of the

isolates against other test organisms, especially those that are known to cause diseases, is also recommended.

\section{References}

1. World Health Organization (2016) Antimicrobial resistance.

2. Falagas M, Grammatikos A, Michalopoulos A (2014) Potential of oldgeneration antibiotics to address current need for new antibiotics. Expert Rev Anti-infec Thera 6: 593-600.

3. Spížek J, Novotná J, Řezanka T, Demain AL (2010) Do we need new antibiotics? The search for new targets and new compounds. J Indus Microbiol Biotechnol 37: 1241-1248.

4. Moloney MG (2016) Natural products as a source of novel antibiotics. Trends Pharmacol Sci 37: 689-701.

5. Quintana J, Vanegas JB, Costa GM, Castellanos L, Arevalo C (2015) Marine organisms as source of extracts to disrupt bacterial communication: Bioguided isolation and identification of quorum sensing inhibitors from Ircinia felix. Revista Brasileira de Farmacognosia 25: 199-207.

6. Mehbub MF, Lei J, Franco C, Zhang W (2014) Marine sponge derived natural products between 2001 and 2010: Trends and opportunities for discovery of bioactives. Nat Center Biotechnol Inform 12: 4539-4577.

7. Laport M, Santos O (2009) Marine sponges: potential sources of new antimicrobial drugs. Current Pharmaceu Biotechnol 10: 86-105.

8. Calado R, Gomes NC, Leal MC, Puga J, Serôdio J (2012) Trends in the discovery of new marine natural products from invertebrates over the last two decades-where and what are we bioprospecting? PLoS ONE 7: e30580.

9. Indraningrat AAG, Smidt H, Sipkema D (2016) Bioprospecting spongeassociated microbes for antimicrobial compounds. Marine Drug 14: 87.

10. Anand TP, Bhat AW, Roy U, Sarma SP, Shouche YS (2006) Antimicrobial activity of marine bacteria associated with sponges from the waters off the coast of South East India. Microbiol Res 161: 252-262.

11. Thomas TRA, Kavlekar DP, Loka Bharathi PA (2010) Marine drugs from sponge-microbe association-A Review. Marine Drug 8: 1417-1468.

12. Pastoriza LR, Sigod JO (2005) Antibacterial activity of the crude extract from an unidentified marine sponge collected from White Island Islet, Illana Bay. Marine Drud 9: 1451-1456.

13. Hausmann R, Vitello MP, Leitermann F, Syldatk C (2006) Advances in the production of sponge biomass Aplysina aerophoba-a model sponge for ex situ sponge biomass production. J Biotechnol 124: 117-127.

14. Dingal PCD (2005) Bioassay-guided fractionation of Thorecta sp. From Pagahan, Initao, Misamis Oriental. J Biotechnol 112: 125-128.

15. Abad MJ, Bedoya LM, Bermejo P (2011) Marine compounds and their antimicrobial activities. Communic Curr Res Technol Adv 51: 1293-1306.

16. Datta D, Talapatra SN, Swarnakar S (2015) Bioactive compounds from marine invertebrates for potential medicines-An overview. Interna Lett Nat Sci 34: 42-61.

17. Hutagalung RA, Victor Karjadidjaja M, Prasasty VD, Mulyono N (2014) Extraction and characterization of bioactive compounds from cultured and natural sponge, Haliclona molitba and Stylotella aurantium origin of Indonesia. Intern J Biosci Biochem Bioinfor 4: 302.

18. Kim SK, Dewapriya P (2012) Bioactive compounds from marine sponges and their symbiotic microbes: A potential source of nutraceuticals. Adv Food Nutri Res 65: 137-151.

19. Sinko J, Rajchard J, Balounova Z, Fikotova L (2012) Biologically active substances from water invertebrates: A Review. Veteri Medic 57: 177-184.

20. Said SA, Moshi MJ, Nondo RSO, Masimba PJ, Innocent E (2011) Evaluation of the potential of the marine sponges of the Zanzibar Island $t$ o yield antimalarial and antimicrobial active compounds. Tanzania J Heal Res 12: 195-202.

21. Soulange JG, Marie D, Kauroo S, Beesoo R, Ramanjooloo A (2014) Antibacterial properties of marine sponges from Mauritius waters. Tropical J Pharmaceu Res 13: 249-254. 


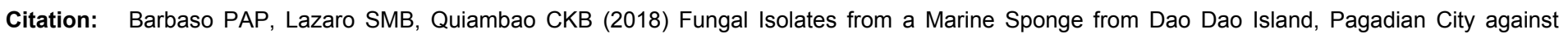

Bacillus subtilis, Microccocus luteus, Rhizopus oryzae, and Saccharomyces cerevisiae. Nat Prod Chem Res 6: 309. doi: 10.4172/2329-6836.1000309

Page 10 of 10

22. Guantai A, Innocent E, Masimba PJ, Moshi MJ, Nondo RS et al. (2010) Evaluation of the potential of the marine sponges of the Zanzibar Island to yield antimalarial and antimicrobial active compounds. Tanzania J Heal Res 12: 195-202.

23. Bado SMC (2006) Antibiotic producing microbial isolates associated with some sponges in Mantigue Islet, Camiguin.

24. Radu S, Kqueen CY (2002) Preliminary screening of endophytic fungi from medicinal plants in Malaysia for antimicrobial and antitumor activity. The Malaysian J Med Sci 9: 23-33.

25. HiMedia Laboratories (2015) YPG agar. HiMedia.

26. http://www.tagmydoc.com/viewer?url=http://www.tagmydoc.com/dl/ QzAxq/6vs
27. Oclarit JM, Okada H, Ohta S, Kamimura K, Yamaoka Y et al. (1994) Production of antibacterial agent, o-amiphenol by a bacterium isolated from the marine sponge, Adocia sp. Fisher Sci 60: 559-562.

28. Mustanir Y, Hindiah MH, Murniana H (2014) Antibacterial activities of ethyl acetate extract from Plumeria alba stem bark. Scien Study Res 15: 107-112.

29. Kelley K, Lai K, Wu P (2008) Using R for data analysis. A Best Practice for Research, pp: 535-572.

30. Hammer $\varnothing$, Harper DA, Ryan PD (2001) PAST: Paleontological statistics software package for education and data analysis. Paleontologia Electronica 4: 9. 